\title{
Tentative detection of phosphine in IRC $+10216^{\star}$
}

\author{
M. Agúndez ${ }^{1}$, J. Cernicharo ${ }^{1}$, J. R. Pardo ${ }^{1}$, M. Guélin² ${ }^{2}$ and T. G. Phillips ${ }^{3}$ \\ 1 Departamento de Astrofísica Molecular e Infrarroja, Instituto de Estructura de la Materia, CSIC, Serrano 121, 28006 Madrid, Spain \\ e-mail: [marce; cerni; pardo]@damir.iem.csic.es \\ 2 Institut de Radioastronomie Millimétrique, 300 rue de la Piscine, 38406 St. Martin d'Hères, and LERMA/École Normale \\ Supérieure, 24 rue Lhomond, 75231 Paris, France \\ e-mail: guelin@iram.fr \\ ${ }^{3}$ California Institute of Technology, Downs Laboratory of Physics 320-47, Pasadena, CA 91125, USA \\ e-mail: tgp@submm.caltech.edu
}

Received 14 May 2008 / Accepted 27 May 2008

\section{ABSTRACT}

\begin{abstract}
Aims. The $J_{\mathrm{K}}=1_{0}-0_{0}$ rotational transition of phosphine $\left(\mathrm{PH}_{3}\right)$ at $267 \mathrm{GHz}$ has been tentatively identified with a $T_{\mathrm{MB}} \sim 40 \mathrm{mK}$ spectral line observed with the IRAM 30-m telescope in the C-star envelope IRC +10216

Methods. A radiative transfer model was used to fit the observed line profile.

Results. The derived $\mathrm{PH}_{3}$ abundance relative to $\mathrm{H}_{2}$ is $6 \times 10^{-9}$, although it may have a large uncertainty due to the lack of knowledge about the spatial distribution of this species. If our identification is correct, it implies that $\mathrm{PH}_{3}$ has a similar abundance to what is reported for $\mathrm{HCP}$ in this source and that these two molecules $\left(\mathrm{HCP}\right.$ and $\left.\mathrm{PH}_{3}\right)$ together take up about $5 \%$ of phosphorus in IRC +10216 . The abundance of $\mathrm{PH}_{3}$, like that of other hydrides in this source, is not well explained by conventional gas-phase LTE and non-LTE chemical models, and may imply formation on grain surfaces.
\end{abstract}

Key words. stars: individual: IRC +10216 - stars: carbon - radio lines: stars - astrochemistry - line: identification stars: AGB and post-AGB

\section{Introduction}

Of the nearly 150 molecules detected so far in interstellar and circumstellar media, around $3 / 4$ can be formed from just four elements $(\mathrm{H}, \mathrm{C}, \mathrm{N}$, and $\mathrm{O})$. The remaining $1 / 4$ contain metals $(\mathrm{Na}, \mathrm{K}, \mathrm{Al}, \mathrm{Mg}$, and $\mathrm{Fe})$, halogens $(\mathrm{F}$ and $\mathrm{Cl})$, and to a large extent the second-row elements $\mathrm{Si}, \mathrm{P}$, and $\mathrm{S}$. The scarcity of molecules containing the second-row elements $\mathrm{Si}, \mathrm{P}$, and $\mathrm{S}$, compared to their first-row analogs $\mathrm{C}, \mathrm{N}$, and $\mathrm{O}$, on one hand, reflects a lower cosmic abundance $(\mathrm{Si} / \mathrm{C} \sim 1 / 8, \mathrm{P} / \mathrm{N} \sim 1 / 300$, and $\mathrm{S} / \mathrm{O} \sim$ 1/30; Asplund et al. 2005) and, on the other, important chemical differences. Second-row elements have generally a larger refractory character; i.e., they tend to form solid condensates and deplete from the gas phase (Field 1974; Turner 1991; Lodders \& Fegley 1999). Also, chemical bonds formed by first-row elements are generally stronger and the resulting molecules are stabler than the corresponding second-row analogs. For example, the high stability of $\mathrm{C}-\mathrm{C}$ bonds allows a wide variety of organic molecules to form, while an Si-based chemistry is much more limited.

The very limited number of interstellar and circumstellar P-bearing molecules known for a long time, just PN and CP (Turner \& Bally 1987; Ziurys 1987; Guélin et al. 1990) in contrast to the nearly $50 \mathrm{~N}$-bearing molecules found, may have been related to a sort of mixing of all the factors mentioned

\footnotetext{
* Based on observations carried out with the IRAM 30-m telescope and the Caltech Submillimeter Observatory (CSO). IRAM is supported by INSU/CNRS (France), MPG (Germany), and IGN (Spain). The CSO is operated by the California Institute of Technology under funding from the National Science Foundation, Grant No. AST-0540882.
}

above: first, the more than hundred-fold decrease in the cosmic abundance of $\mathrm{P}$ compared to that of $\mathrm{N}$; second, the likely strong depletion of phosphorus suggested by observations of some P-bearing species (Turner et al. 1990); and lastly, some important differences between the chemistry of $\mathrm{P}$ and that of $\mathrm{N}$, which are indicated by, for example, the non reactivity of $\mathrm{PH}_{n}^{+}$ $(n=0,3)$ ions with $\mathrm{H}_{2}$ as opposed to the case of $\mathrm{NH}_{n}^{+}$(Thorne et al. 1984; Adams et al. 1990).

In recent years, however, we have seen the discoveries, mostly in circumstellar media, of new phosphorus compounds such as HCP, PO, and $\mathrm{C}_{2} \mathrm{P}$ (Agúndez et al. 2007; Milam et al. 2008; Tenenbaum et al. 2007; Halfen et al. 2008), which permit us to discuss the chemistry of phosphorus on a wider observational basis. In this letter we present the tentative detection of $\mathrm{PH}_{3}$, the phosphorus analog of $\mathrm{NH}_{3}$, in the carbon-rich circumstellar envelope IRC +10216 . This species, known to be abundant in the atmospheres of the giant gaseous planets Jupiter and Saturn (Weisstein \& Serabyn 1996), has never been observed outside the Solar System.

\section{Observations and results}

The phosphine molecule, $\mathrm{PH}_{3}$, is an oblate symmetric top, thus its rotational levels are given by two quantum numbers $(J, K)$, and radiative transitions are only allowed within levels of the same $K$ ladder $(\Delta J=1, \Delta K=0)$. The $K$ ladders are grouped into two distinct forms: ortho $(K=3 n, n$ an integer) and para $(K \neq$ $3 n$ ), between which both radiative and collisional transitions are severely forbidden. Its rotational spectrum has been extensively investigated in the laboratory, allowing for the very weak 
"forbidden" transitions $(\Delta J=0, \Delta K= \pm 3$ ) to be measured and for the hyperfine structure due to the ${ }^{1} \mathrm{H}$ and the ${ }^{31} \mathrm{P}$ nuclear spins to be resolved (see Cazzoli \& Puzzarini 2006, and references therein). In contrast to $\mathrm{NH}_{3}$, no evidence of inversion doubling has been found in the case of $\mathrm{PH}_{3}$. The electric dipole moment has been measured as $0.57395 \pm 0.0003 \mathrm{D}$ (Davies et al. 1971).

Following the recent detection of HCP in IRC +10216 (Agúndez et al. 2007), it was speculated that $\mathrm{PH}_{3}$ might be detectable in this source if the $\mathrm{PH}_{3} / \mathrm{HCP}$ abundance ratio is similar to the $\mathrm{NH}_{3} / \mathrm{HCN}$ one. Prompted by this hypothesis we searched for the fundamental ortho- $\mathrm{PH}_{3} 1_{0}-0_{0}$ line, at $267 \mathrm{GHz}$, with the IRAM 30-m telescope. Preliminary observations with a low spectral resolution, 1.25 MHz, were done in 2007 May and we found significant emission at the frequency of the $\mathrm{PH}_{3} 1_{0}-0_{0}$ line and with the expected linewidth $\left(v_{\exp }=14.5 \mathrm{~km} \mathrm{~s}^{-1}\right.$ for most of the molecular lines in IRC +10216; Cernicharo et al. 2000). The line, however, showed a profile unusual for IRC +10216 : neither really U-shaped nor flat-topped. Encouraged by this result we returned to the 30-m telescope in 2008 February and April to observe this transition again with 4 times higher spectral resolution $(320 \mathrm{kHz})$. Two SIS receivers operating at $1 \mathrm{~mm}$ were used simultaneously with upper sideband rejections of $\sim 10 \mathrm{~dB}$. The local oscillator was shifted by $80 \mathrm{MHz}$ to identify any contribution from the image sideband. An autocorrelator was used as backend to provide the required spectral resolution of $320 \mathrm{kHz}$. The secondary mirror was wobbled by $\pm 90^{\prime \prime}$ at a rate of $0.5 \mathrm{~Hz}$. The pointing and focus of the telescope were checked every $1-2 \mathrm{~h}$ on Saturn, which was closer than $10^{\circ}$ to IRC +10216 . Here we express the intensity scale in units of main beam brightness temperature $T_{\mathrm{MB}}$. The parameter $B_{\text {eff }} / F_{\text {eff }}$, used to convert $T_{\mathrm{A}}^{*}$ into $T_{\mathrm{MB}}$, is 0.51 and the beam size is $9^{\prime \prime}$ at $267 \mathrm{GHz}$ for the $30-\mathrm{m}$.

The resulting spectrum at $267 \mathrm{GHz}$ is shown at the top of Fig. 1. The line profile indicates the blend of a normal cusped line of width $\sim 29 \mathrm{~km} \mathrm{~s}^{-1}$, characteristic of optically thin lines arising in the outer envelope (e.g. $\mathrm{C}_{2} \mathrm{H}$ ), with a narrower $\left(\sim 6 \mathrm{~km} \mathrm{~s}^{-1}\right)$ sharply peaked line, characteristic of vibrationally excited lines arising close to the star. This latter line was soon identified as the $J=15-14$ transition of SiS in its $v=4$ vibrational state, based on SiS laboratory spectroscopic data (Sanz et al. 2003; Müller et al. 2007). Emission of SiS in vibrationally excited states up to $v=3$ has been reported toward IRC +10216 (Turner 1987; Fonfría et al. 2006; Cernicharo et al. 2000). The linewidths of vibrationally excited $\mathrm{SiS}$ are unusually narrow implying that the emission arises from the innermost envelope where the gas has not yet reached the terminal expansion velocity of $14.5 \mathrm{~km} \mathrm{~s}^{-1}$. To constrain the possible contribution of the $J=15-14$ line of SiS $v=4$ to the $267 \mathrm{GHz}$ line, we observed the immediate previous $J$ transitions. In Fig. 1 we show the $J=14-13$ and $J=13-12$ lines of SiS in the $v=4$ state (both observed with a spectral resolution of $320 \mathrm{kHz}$ at the time of the $267 \mathrm{GHz}$ observations) and the $J=12-11$ line (observed in a previous run in 2005 January with a spectral resolution of $1.25 \mathrm{MHz}$ ).

Except for the $J=14-13$ line of $\operatorname{SiS} v=4$, which is partially blended with a fine structure component of the $N=27-26$ transition of $\mathrm{CCC}^{13} \mathrm{CH}$, the observations clearly show that $\mathrm{SiS} v=$ 4 lines are narrow, with expansion velocities of about $3 \mathrm{~km} \mathrm{~s}^{-1}$ (see Table 1). With this in mind, the observed emission feature at $267 \mathrm{GHz}$ has been fitted by two line components, a narrow one corresponding to the $J=15-14$ transition of SiS $v=4$ and a wider line whose width, $v_{\exp }=14.1 \pm 0.3 \mathrm{~km} \mathrm{~s}^{-1}$, agrees with the expansion velocity of $14.5 \mathrm{~km} \mathrm{~s}^{-1}$ in IRC +10216 , and whose center rest frequency, $266944.5 \pm 0.3 \mathrm{MHz}$, is in very good agreement with the laboratory frequency of the $1_{0}-0_{0}$ line

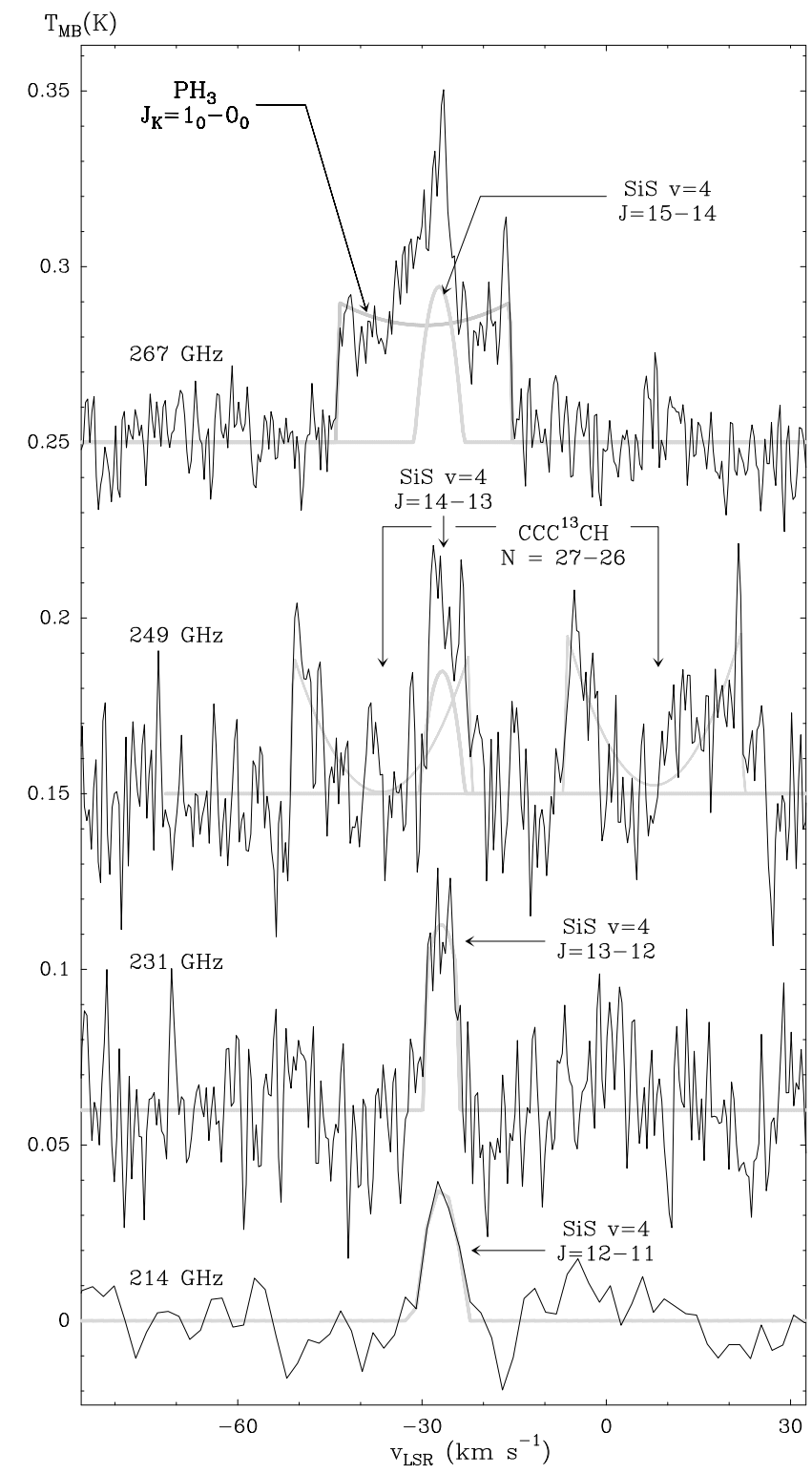

Fig. 1. Spectra of IRC +10216 observed with the IRAM 30-m telescope showing on top the $J_{\mathrm{K}}=1_{0}-0_{0}$ line of $\mathrm{PH}_{3}$ blended with a narrower line assigned to the $J=15-14$ line of $\operatorname{SiS} v=4$. Shown below are the immediate lower rotational transitions of $\operatorname{SiS} v=4$. Fits to the line profiles using the CLASS shell method are shown as thick grey lines.

of $\mathrm{PH}_{3}$ (see Table 1). The hyperfine structure is not resolved as the components are separated by less than $0.2 \mathrm{~km} \mathrm{~s}^{-1}$ in velocity (Cazzoli \& Puzzarini 2006), which is lower than the spectral resolution. The good agreement between the observed and laboratory frequencies is the strongest evidence of $\mathrm{PH}_{3}$ detection in IRC +10216

Besides the $\operatorname{SiS} v=4$ line, there are some other lines with frequencies close to that of $\mathrm{PH}_{3} 1_{0}-0_{0}$. Most of them, such as $\mathrm{SO}_{2}$ $30_{9,21}-31_{8,24}$ at $266943.344 \mathrm{MHz}$ or $\mathrm{CH}_{3} \mathrm{CH}_{2} \mathrm{CN} 15_{4,12}-15_{2,13}$ at $266951.639 \mathrm{MHz}$, are ruled out as likely contributors since many other lines of these species should have been detected. A more plausible species is $\mathrm{HC}_{3} \mathrm{~N}$ in the $v_{7}=4$ vibrational state, whose transition $J=29-28 \ell=0$ e lies at $266943.313 \mathrm{MHz}$ (Mbosei et al. 2000). Although several lines of $\mathrm{HC}_{3} \mathrm{~N}$ in the vibrational excited state $v_{7}=1$ have been observed in IRC +10216 (Cernicharo et al. 2000), we rule out that $\mathrm{HC}_{3} \mathrm{~N} v_{7}=4$ is the main contributor to the $267 \mathrm{GHz}$ emission based on the 
Table 1. Observed line parameters in IRC +10216 .

\begin{tabular}{lcccc}
\hline \hline Transition & $\begin{array}{c}\text { Cal. Freq. } \\
(\mathrm{MHz})\end{array}$ & $\begin{array}{c}\text { Obs. Freq. } \\
(\mathrm{MHz})\end{array}$ & $\begin{array}{c}v_{\mathrm{exp}}{ }^{a} \\
\left(\mathrm{~km} \mathrm{~s}^{-1}\right)\end{array}$ & $\begin{array}{c}\int T_{\mathrm{MB}} \mathrm{d} v \\
\left(\mathrm{~K} \mathrm{~km} \mathrm{~s}^{-1}\right)\end{array}$ \\
\hline $\mathrm{PH}_{3}$ & & & & \\
$J_{\mathrm{K}}=1_{0}-0_{0}$ & 266944.514 & $266944.5(3)$ & $14.1(3)$ & $1.002(40)$ \\
\hline $\mathrm{SiS} v=4$ & & & & \\
$J=15-14$ & 266941.754 & $266942.4(7)$ & $4.0(7)$ & $0.237(40)$ \\
$J=14-13$ & 249155.372 & $249155.6(4)$ & $3.5(4)$ & $0.172(40)$ \\
$J=13-12$ & 231366.976 & $231367.2(3)$ & $2.7(3)$ & $0.256(30)$ \\
$J=12-11$ & 213576.710 & $213576.9(5)$ & $3.5(5)$ & $0.207(40)$ \\
\hline
\end{tabular}

NOTE. Number in parentheses are $1 \sigma$ uncertainties in units of the last digits. Observed frequencies are given in the rest frame of IRC +10216 assuming a systemic velocity of $v_{\mathrm{LSR}}=-26.5 \mathrm{~km} \mathrm{~s}^{-1}$.

${ }^{a} v_{\exp }$ is the half width at zero level.

upper limit of $T_{\mathrm{MB}}<0.01 \mathrm{~K}$ that we have from our $30-\mathrm{m}$ data archive for the lower- $J$ transition $J=26-25 \ell=0$ e transition at $239370.171 \mathrm{MHz}$.

We tried to confirm the identification of $\mathrm{PH}_{3}$ by observing other transitions. The $J=2-1$ line at $534 \mathrm{GHz}$ is not reachable from the ground due to severe atmospheric absorption. We, thus, searched for the $J=3-2$ transition at $800 \mathrm{GHz}$ with the Caltech Submillimeter Observatory (CSO). The observations were carried out in 2008 January using the chopping secondary mode with a throw of $\pm 90^{\prime \prime}$ at a rate of $1.2 \mathrm{~Hz}$. The SIS receiver was tuned in double sideband and a Fast Fourier Transform Spectrometer was used as backend to provide a spectral resolution of $0.12 \mathrm{MHz}$. The pointing of the telescope was checked on Saturn. The beam size of the CSO at $800 \mathrm{GHz}$ is $11.5^{\prime \prime}$ and the beam efficiency is 0.28 . In spite of the good atmospheric conditions (zenith sky opacity at $225 \mathrm{GHz}$ was $0.04-0.08$ ), the high opacity of the atmosphere at $800 \mathrm{GHz}\left(T_{\text {sys }}\right.$ ranged from $3000 \mathrm{~K}$ to $8000 \mathrm{~K}$ ) did not allow us to reach a low enough noise level to confirm or discard the presence of $\mathrm{PH}_{3}$ (see top panel in Fig. 2).

\section{Analysis and discussion}

To interpret the observations, we computed line profiles by means of excitation and radiative transfer calculations based in the large velocity gradient (LVG) formalism (Castor 1970), coupled to the spectral catalog of J. Cernicharo (Cernicharo et al. 2000). We consider separately both the ortho (o- $\left.\mathrm{PH}_{3}\right)$ and para $\left(\mathrm{p}-\mathrm{PH}_{3}\right)$ species of phosphine (the ortho-to-para ratio was assumed to be 1 , the statistical value). The energy levels and transition frequencies were computed from the rotational constants reported by Cazzoli \& Puzzarini (2006). We included rotational levels in the ground vibrational state up to $J_{\mathrm{K}}=7_{6}$ for o- $\mathrm{PH}_{3}$ and up to $J_{K}=5_{5}$ for $\mathrm{p}-\mathrm{PH}_{3}$. As rate coefficients for collisional de-excitation of $\mathrm{o}-\mathrm{PH}_{3}\left(\mathrm{p}-\mathrm{PH}_{3}\right)$ with $\mathrm{H}_{2}$ and $\mathrm{He}$, we adopted those computed for collisions of $\mathrm{o}-\mathrm{NH}_{3}\left(\mathrm{p}-\mathrm{NH}_{3}\right)$ with $\mathrm{p}-\mathrm{H}_{2}$ (Danby et al. 1988) and He (Machin \& Roueff 2005) respectively, properly corrected to the case in which inversion doubling is not resolved.

We assumed a distance to IRC +10216 of $150 \mathrm{pc}$ and simulated the circumstellar envelope as a spherically distributed gas expanding at a constant velocity of $14.5 \mathrm{~km} \mathrm{~s}^{-1}$. The gas density and temperature radial profiles were taken from Agúndez \& Cernicharo (2006). Phosphine was assumed to be distributed between an inner radius $R_{\text {in }}$ and an outer radius $R_{\text {out }}$, with a constant abundance, $x\left(\mathrm{PH}_{3}\right)$, relative to $\mathrm{H}_{2}$. We took $R_{\text {in }}=1.3 \times$ $10^{15} \mathrm{~cm}$ (about 20 stellar radii), the value adopted by Hasegawa et al. (2006) for $\mathrm{NH}_{3}$. The values of $R_{\text {out }}$ and $x\left(\mathrm{PH}_{3}\right)$ were varied
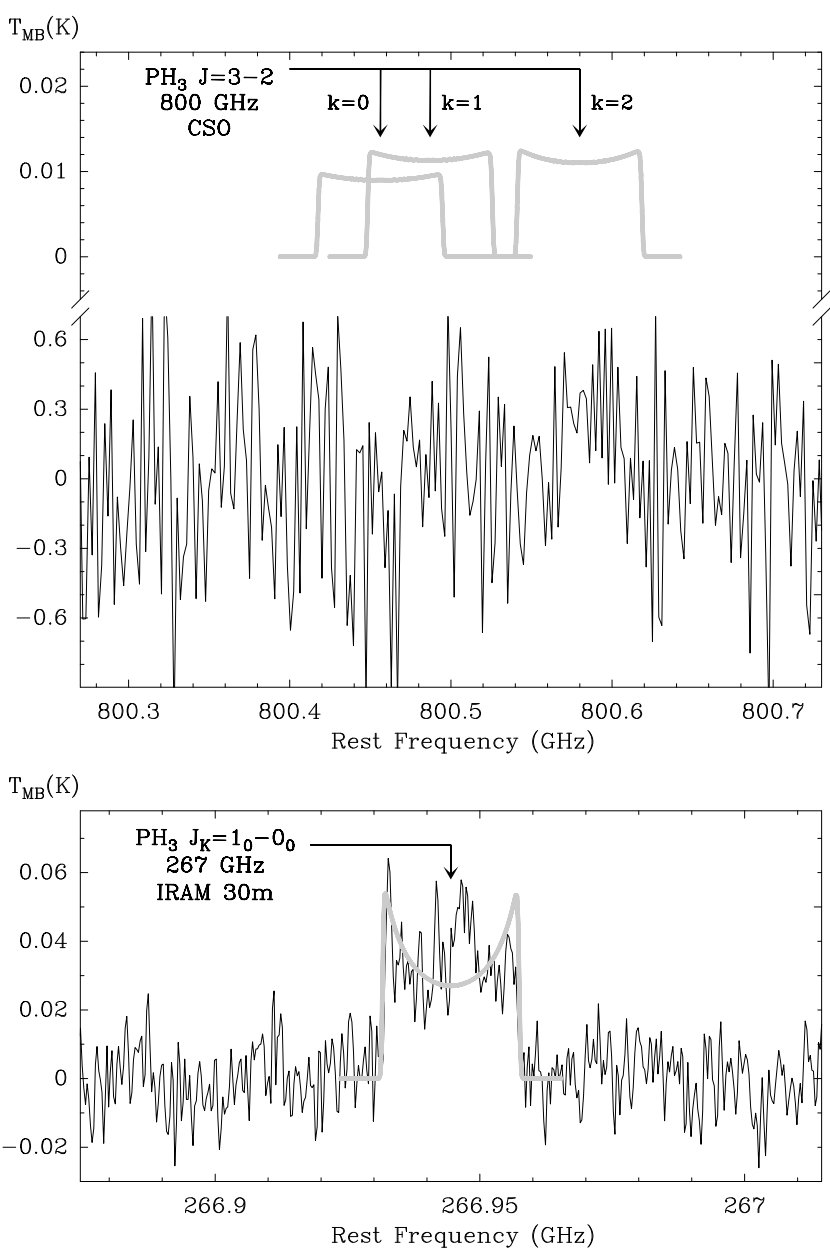

Fig. 2. The lower panel shows the $\mathrm{PH}_{3} J_{\mathrm{K}}=1_{0}-0_{0}$ line at $267 \mathrm{GHz}$ as observed with the IRAM 30-m telescope, in which the fit to the SiS $v=$ $4 J=15-14$ line has been subtracted. The upper panel shows the observation at $800 \mathrm{GHz}$ with the CSO smoothed to a spectral resolution of $2 \mathrm{MHz}$. The thick grey lines in both panels correspond to the line profiles given by the LVG model.

until obtaining the best fit to the observed $\mathrm{PH}_{3} 1_{0}-0_{0}$ line profile. Since both gas density and temperature vary greatly with radius, we divided the envelope into various shells and solved for the level populations in each shell independently of the others. We then computed the emergent intensity and weighted it with the main beam of the selected telescope.

In the bottom panel of Fig. 2 we plot the observed $1_{0}-0_{0}$ $\mathrm{PH}_{3}$ line profile, obtained by subtracting the fit to the $\operatorname{SiS} v=$ 4 blended line. Also shown is the profile resulting from our best LVG model, whose parameters are $R_{\text {out }}=2 \times 10^{16} \mathrm{~cm}$ and $x\left(\mathrm{PH}_{3}\right)=6 \times 10^{-9}$. The model indicates that the upper level involved in the $1_{0}-0_{0} \mathrm{PH}_{3}$ line is mostly populated by collisions in the inner $4 \times 10^{15} \mathrm{~cm}$, which corresponds to an angular diameter of about $4^{\prime \prime}$ and is thus spatially diluted in the $9^{\prime \prime}$ beam of the $30-\mathrm{m}$ telescope. The top panel of Fig. 2 shows the corresponding prediction for the $\mathrm{PH}_{3} J=3-2$ line profiles as observed with the CSO. It is seen that the expected intensity is well below the noise of the observed spectrum and is thus consistent with the IRAM 30-m result for the $J=1-0$ line. The spatial dilution is also important for the CSO observations and plays against our efforts to detect the $J=3-2$ lines.

It should be noted that the derived abundance and distribution of $\mathrm{PH}_{3}$ are very uncertain. Since we observed just one 
single $\mathrm{PH}_{3}$ line, a considerable degeneracy exists between models with different values of $R_{\text {in }}, R_{\text {out }}$, and $x\left(\mathrm{PH}_{3}\right)$. Moreover, infrared pumping to excited vibrational states, not considered in our model, may play an important role in the excitation of the rotational levels in the ground vibrational state. Phosphine has indeed many vibrational bands in the spectral region around $10 \mu \mathrm{m}$, a wavelength at which the central source has its maximum flux (Cernicharo et al. 1999).

Confirmation of our tentative detection may rely on further observations of other $\mathrm{PH}_{3}$ transitions. The $J=2-1$ and $J=3-2$ lines at $534 \mathrm{GHz}$ and $800 \mathrm{GHz}$, respectively, are observable with the Herschel Space Observatory (HSO), although our LVG model predicts somewhat weaker intensities than with the CSO due to the stronger dilution effect. In the case of the Atacama Large Millimeter Array (ALMA), the high angular resolution that it will provide, better than $3^{\prime \prime}$ at $800 \mathrm{GHz}$, will perfectly fit with the expected size of the $J=3-2$ emission. The predictions indicate main beam brightness temperatures of about $1 \mathrm{~K}$, which provide a good opportunity for detecting it in spite of the high atmospheric opacity at this frequency. We note that these predictions are based on our best LVG model, but the $J=$ 2-1 and $J=3-2$ lines could be more intense than expected if infrared pumping is playing an important role, or if $\mathrm{PH}_{3}$ is present in hot regions closer to the star than the inner cutoff of $1.3 \times$ $10^{15} \mathrm{~cm}$ assumed here.

The derived abundance of $\mathrm{PH}_{3}, x\left(\mathrm{PH}_{3}\right)=6 \times 10^{-9}$, implies that it takes up about $1.3 \%$ of the available phosphorus. The $\mathrm{PH}_{3} / \mathrm{HCP}$ ratio is then found to be $1 / 2.3$ (Agúndez et al. 2007), noticeably higher than the $\mathrm{NH}_{3} / \mathrm{HCN}$ ratio, which is about $1 / 50$ in IRC +10216 (Hasegawa et al. 2006; Fonfría et al. 2007). The formation of $\mathrm{PH}_{3}$ in the gas phase is difficult to explain. Its LTE abundance in the inner envelope is rather low, $<10^{-12}$, and gas phase reactions yield no net formation in the outer envelope (Agúndez et al. 2007). Besides $\mathrm{PH}_{3}$, other hydrides such as $\mathrm{NH}_{3}$, $\mathrm{CH}_{4}, \mathrm{H}_{2} \mathrm{O}, \mathrm{SiH}_{4}$, and $\mathrm{H}_{2} \mathrm{~S}$ are observed in IRC +10216 with relatively high abundances (Keady \& Ridgway 1993; Hasegawa et al. 2006; Agúndez \& Cernicharo 2006). Many of them are usually assumed as parent molecules, i.e. formed in the inner envelope, in most chemical models of IRC +10216 (e.g. MacKay \& Charnley 2001; Agúndez \& Cernicharo 2006). However, chemical equilibrium calculations, similar to those reported in Agúndez et al. (2007), indicate much lower abundances than observed, except perhaps for $\mathrm{CH}_{4}$. A widely invoked explanation, when gas phase chemistry fails to explain an observed abundance, is that of grain surface reactions. In the case of hydrides such as $\mathrm{PH}_{3}$, a likely formation process is the direct hydrogenation of the heavy atom taking place on grain surfaces.

\section{Conclusions}

We have tentatively detected $\mathrm{PH}_{3}$ in IRC +10216 through its $J_{\mathrm{K}}=1_{0}-0_{0}$ transition at $267 \mathrm{GHz}$. The derived abundance relative to $\mathrm{H}_{2}$ is $6 \times 10^{-9}$. Despite considerable uncertainty, this value is similar to the HCP abundance found in this source (Agúndez et al. 2007). These two species, $\mathrm{HCP}$ and $\mathrm{PH}_{3}$, would then take up about $5 \%$ of the phosphorus in IRC +10216 . The formation of $\mathrm{PH}_{3}$, unlike that of $\mathrm{HCP}$, is difficult to explain in the gas phase and could occur on grain surfaces. It remains a target for the future to confirm this tentative detection by observing the $J=3-2$ transition, at $800 \mathrm{GHz}$, with the ALMA facility. Also, further observations at $267 \mathrm{GHz}$ in other sources such as CRL 2688, where HCP has been also detected (Milam et al. 2008), will be of great interest to support this tentative detection and to understand the chemistry behind it.

Acknowledgements. We thank the IRAM staff, especially C. Thum, for their kindness during the 30-m observations. We also acknowledge funding support from Spanish MEC trough grants AYA2006-14876 and ESP2004-665, and from Spanish CAM under PRICIT project S-0505/ESP-0237 (ASTROCAM). M.A. also acknowledges grant AP2003-4619 from Spanish MEC.

Note added in proof. We have very recently been aware that E. D. Tenenbaum and L. M. Ziurys (ApJL, in press) have observed with the Arizona Radio Observatory (ARO) Submillimeter Telescope (SMT) the same emission line at $267 \mathrm{GHz}$, which they also interpret as the $J_{K}=1_{0}-0_{0}$ transition of $\mathrm{PH}_{3}$, in IRC +10216 and also in CRL 2688.

\section{References}

Adams, N. G., McIntosh, B. J., \& Smith, D. 1990, A\&A, 232, 443

Agúndez, M., \& Cernicharo, J. 2006, ApJ, 650, 374

Agúndez, M., Cernicharo, J., \& Guélin, M. 2007, ApJ, 662, L91

Asplund, M., Grevesse, N., \& Sauval, A. J. 2005, in Cosmic Abundances as Records of Stellar Evolution and Nucleosynthesis, ed. T. G. Barnes, III, \& F. N. Bash (San Francisco: ASP), ASP Conf. Ser., 336, 25

Castor, J. I. 1970, MNRAS, 149, 111

Cazzoli, G., \& Puzzarini, C. 2006, J. Mol. Spectr., 239, 64

Cernicharo, J., Yamamura, I., González-Alfonso, E., et al. 1999, ApJ, 526, L41 Cernicharo, J., Guélin, M., \& Kahane, C. 2000, A\&AS, 142, 181

Danby, G., Flower, D. R., Valiron, P., et al. 1988, MNRAS, 235, 229

Davies, P. B., Neumann, R. M., Wofsy, S. C., \& Klemperer, W. 1971, J. Chem. Phys., 55, 3564

Field, G. B. 1974, ApJ, 187, 453

Fonfría, J. P., Agúndez, M., Tercero, B., et al. 2006, ApJ, 646, L127

Fonfría, J. P., Cernicharo, J., Richter, M. J., \& Lacy, J. 2007, ApJ, 673, 445

Guélin, M., Cernicharo, J., Paubert, G., \& Turner, B. E. 1990, A\&A, 230, L9

Halfen, D. T., Clouthier, D. J., \& Ziurys, L. M. 2008, ApJ, 677, L101

Hasegawa, T. I., Kwok, S., Koning, N., et al. 2006, ApJ, 637, 791

Keady, J. J., \& Ridgway, S. T. 1993, ApJ, 406, 199

Lodders, K., \& Fegley, B. Jr. 1999, in Asymptotic Giant Branch Stars, ed. T. Le Bertre, A. Lèbre, \& C. Waelkens (San Francisco: ASP), IAU Symp., 191, 279

Machin, L., \& Roueff, E. 2005, J. Phys. B: Mol. Opt. Phys., 38, 1519

MacKay, D. D. S., \& Charnley, S. B. 2001, MNRAS, 325, 545

Mbosei, L., Fayt, A., Dréan, P., \& Cosléou, J. 2000, J. Mol. Struct., 517, 271

Milam, S. N., Halfen, D. T., Tenenbaum, E. D., et al. 2008, ApJ, in press

Müller, H. S. P., McCarthy, M. C., Bizzocchi, L., et al. 2007, Phys. Chem. Chem. Phys., 9, 1579

Sanz, M. E., McCarthy, M. C., \& Thaddeus, P. 2003, J. Chem. Phys., 119, 11715

Tenenbaum, E. D., Woolf, N. J., \& Ziurys, L. M. 2007, ApJ, 666, L29

Thorne, L. R., Anicich, V. G., Prasad, S. S., \& Huntress, W. T. 1984, ApJ, 280, 139

Turner, B. E. 1987, A\&A, 183, L23

Turner, B. E. 1991, ApJ, 376, 573

Turner, B. E., \& Bally, J. 1987, ApJ, 321, L75

Turner, B. E., Tsuji, T., Bally, J., Guélin, M., \& Cernicharo, J. 1990, ApJ, 365, 569

Weisstein, E. W., \& Serabyn, E. 1996, Icarus, 123, 23

Ziurys, L. M. 1987, ApJ, 321, L81 